\title{
Current role of lymphadenectomy in the upper tract urothelial carcinoma
}

\author{
Mario Alvarez-Maestro, Juan Gómez Rivas, Sergio Alonso y Gregorio, Cristina de Castro Guerin, \\ Ángel Tabernero Gómez, Jesús Cisneros Ledo
}

Department of Urology, Hospital Universitario La Paz, Madrid, Spain

Citation: Alvarez-Maestro M, Gómez Rivas J, Alonso y Gregorio S, de Castro Guerin C, Tabernero Gómez Á, Cisneros Ledo J. Current role of lymphadenectomy in the upper tract urothelial carcinoma. Cent European J Urol. 2016; 69: 384-390.

Article history

Submitted: April 11, 2016

Accepted: Sept. 1, 2016

Published online: Nov. 30,

2016
Corresponding author Juan Gómez Rivas Hospital Universitario La Paz 261 Paseo La Castellana 28046 Madrid, Spain phone: +34 661190028 juangomezr@gmail.com
Introduction Lymphadenectomy (LND) has recently attracted considerable interest from urological surgeons, as extended lymphadenectomy might have a role in accurate staging or improving patient survival in those patients with urological malignancies.

Upper tract urothelial carcinoma (UTUC) is a relatively rare neoplasm, accounting for about $5 \%$ of all urothelial cancers. Up to $30 \%$ of patients with muscle-invasive UTUC have metastasis in the regional lymph nodes (LNS), which represents a well-established poor prognostic factor.

Material and methods A medline search was conducted to identify original articles and review articles addressing the role of lymphadenectomy LND in UTUC. Keywords included lymphadenectomy, lymph node excision, nephroureterectomy, and upper tract urothelial carcinoma.

Results LND instead of lymphadenectomy has recently attracted considerable interest from urological surgeons and might have a potential role in improving the oncological outcome in patients with urothelial carcinoma. LND ideally improves disease staging; thereby, we need to find the way to identify the patients who could really benefit from adjuvant systemic theraphy. Template-based LND with Radical Nephroureterectomy (RNU) for high risk disease is gaining support based on accumulating retrospective data and supports its utility as a potentially therapeutic maneuver. RNU is still the gold standard treatment for UTUC, but minimal invasive procedures such as laparoscopic RNU and Robot Assisted Nephroureterectomy (RANU) are becoming more employed in recent years and should be used by expert hands. Conclusions Therapeutic benefits of LND and nodal status on disease free survival (DFS) and Cancer Free Survival (CSS) remains controversial. Although most of the data comes from retrospective studies, we encourage performing well designed, prospective, and multicentre studies to clarify this in the coming years.

Key Words: upper tract urothelial carcinoma 〈〉 lymphadenectomy 〈 nephroureterectomy () overall survival

\section{INTRODUCTION}

Lymphadenectomy (LND) has recently attracted considerable interest from urological surgeons, as extended lymphadenectomy might have a role in accurate staging or improving patient survival in those patients with urological malignancies, including renal cell carcinoma [1], prostate cancer [2], urothelial carcinoma of the bladder [3], and the upper urinary tract [4].

Extended lymphadenectomy LND in renal cell carcinoma might have some benefit in accurate staging in the patients with locally advanced disease, but their therapeutic benefit remains undetermined [5]. In prostate cancer, the therapeutic benefit of extended lymphadenectomy is controversial, despite some advantage in accurate pathological staging [6, 7]. Failure to show the survival benefit of extended lymphadenectomy in renal cell carcinoma or prostate cancer might result from the low incidence of lymphatic metastases of these diseases [7, 8]. Urothelial carcinoma has been known for tumors developing lymphatic metastases at a relatively high incidence of 20-30\% compared with those of other 
urological malignancies $[9,10]$. Thus, lymphadenectomy LND might have a potential role in improving the oncological outcome in patients with urothelial carcinoma compared to those with renal cell carcinoma or prostate cancer.

Upper tract urothelial carcinoma (UTUC) is a relatively rare neoplasm, accounting for about 5\% of all urothelial cancers. Up to $30 \%$ of patients with muscle-invasive UTUC have metastasis in the regional lymph nodes (LNs), which represents a well-established poor prognostic factor, and chemotherapy or radiotherapy rarely results in improvement of patient survival [11]. LNs are the major sites of metastases of UTUC. The practice of a meticulous lymphadenectomy LND for invasive UTUC has not been adopted by urologists worldwide. This is at least in part due to the relatively low incidence of UTUC and, consequently, to an incomplete understanding of the optimal LND template, which varies according to the location of the disease [12]. Thus, the control of nodal involvement is a important issue in the treatment of UTUC. In the present review article, we summarized the current understanding of the role of regional LND in UTUC from the published literature. There are many questions outstanding about LND, so we focused on topics including the boundary of regional LND, its staging, and therapeutic benefit.

\section{MATERIAL AND METHODS}

A medline search was conducted to identify original articles and review articles addressing the role of LND in UTUC. Keywords included lymphadenectomy, lymph node excision, nephroureterectomy, and upper tract urothelial carcinoma.

\section{Historical review lymphadenectomy in upper urinary tract}

Lymphatic metastases are also commonly found in urothelial carcinoma of the upper urinary tract (UCUUT) with an incidence of 30-40\%. Early mapping studies carried out in the 1980s showed that lymphatic spread from tumors of the renal pelvis and upper ureter was primarily to the para-aortic and para-caval nodes, and that from the distal ureter, spread was to the pelvic nodes $[13,14,15]$. Thus, nephroureterectomy with lymphadenectomy was advocated as the standard surgical procedure. In the 1990s, two Japanese studies were published examining the role of lymphadenectomy. Komatsu et al. [16] reported superior patient survival in $\mathrm{pN} 0$, and suggested that lymphadenectomy provided accurate staging of these patients, but the therapeutic benefit is only appli- cable to selected patients. Miyake et al. [17] showed that lymphadenectomy might provide a therapeutic benefit in patients with no lymphovascular invasion. However, a definitive conclusion could not be drawn from these studies because of the small number of patients. On this basis, a growing number of clinical researchers are evaluating the impact of LND in UTUC. Unfortunately, most studies are single-institutions reports based on limited study populations. For this reason, in 2009 a Multi-Institution Collaboration Group, the upper tract urothelial carcinoma collaboration (UTUCC) $[18,19]$, was created to collect data on the nephroureterectomies (RNUs) performed in 13 tertiary hospitals and to investigate the role of LND for staging and therapeutic purposes in UTUC.

\section{Anatomy}

Unfortunately, the anatomy of the lymph drainage of UTUC has not been well studied or described, and templates used from institution to institution have varied widely. This lack of uniformity, coupled with the differing drainage patterns observed depending on where the tumor originates, only muddies the debate. The lymphatic drainage of the kidney/pelvis renal appears to predominantly collect in a predictable pattern, following the blood supply. Kondo et al. [20] retrospectively examined the primary site and incidence of nodal metastases of UTUC (Table 1). The novel findings of this report are that retrocaval nodes are important sites of metastasis for tumors of the right renal pelvis and the upper two thirds of the right ureter, in addition to the hilar and paracaval sites. Interaortocaval nodes are also an important primary site of metastasis for tumors of the upper two thirds of the right ureter. Tumors of the left renal pelvis mainly spread to the renal hilum and para-aortic nodes; tumors of the upper two thirds of the left ureter metastasized into the para-aortic nodes. Finally, tumors of the lower thirds of the ureter on each side tended to spread to pelvic lymph nodes below the aortic bifurcation (common iliac, obturator, and internal iliac nodes). This mapping study was one of the most complete studies to date, but derived from a single-institution retrospective series (limited to a total of 42 patients, of which only 23 were actually confirmed pathologically). LN involvement increases with pathologic stage and grade. For example, one study [21] showed as pathologic $\mathrm{T}$ stage increased from $\mathrm{T} 1, \mathrm{~T} 2$, $\mathrm{T} 3$, to T4, the frequency of nodal involvement was $4.5 \%$, $8.9 \%, 28.7 \%$, and $70.6 \%$, respectively. Various authors recommend varying degrees of aggressiveness from templates, nearly mirroring retroperitoneal lymph node dissection (RPLND) templates for testis 
cancer on one extreme, to less aggressive templates where they only remove hilar nodes.

\section{Role of chemotherapy (neoadjuvant vs. adjuvant) in node-positive patients}

LND ideally improves disease staging, thereby identifying patients who could benefit from adjuvant systemic therapy. Nonetheless, whether improved staging results in improved outcomes depends on the decision to deliver chemotherapy and on the efficacy of the regimen. There are some controversies regarding the prognostic value of tumor location, tumor size, and adjuvant chemotherapy. Although a single study has shown adjuvant chemotherapy (AC) in UTUC might be as effective as neoadjuvant chemotherapy for bladder cancer [22], in a large multi-institutional retrospective study, Hellenthal et al. [23] showed that adjuvant chemotherapy confers minimal impact on overall survival (OS) and cancer specific survival (CSS) in high-risk UTUC patients. The same results were obtained in the subgroup of $\mathrm{pN}+$ patients. Not all patients will be able to receive this treatment because of comorbidities and impaired renal function after radical surgery [24]. Recently, Kim et al. [25] evaluated the efficacy of adjuvant cisplatin-based chemotherapy for locally advanced UTUC (pT3 or pT4 or pT1-2N1-3) following radical nephroureterectomy with bladder cuff resection (RNU) in terms of survival and recurrence. The authors found that adjuvant chemotherapy for locally advanced UTUC can prevent bladder recurrence, but has a minimal effect on cancer-specific survival. Based on these findings, data are currently insufficient to provide evidence of the effectiveness of adjuvant chemotherapy. On the contrary, preoperative chemotherapy followed by aggressive surgical consolidation may yield favourable oncological outcomes in patients with UTUC with loco-regional nodal metastases [26], providing a $14 \%$ rate of complete remission and a significant rate of downstaging [27]. However, there is still an absence of randomized prospective studies to support either neoadjuvant or adjuvant therapy. We should wait for the results of the prospective randomized clinical trial - the POUT [28] (perioperative chemotherapy versus surveillance in upper tract urothelial cancer) trial started in April 2012 in United Kingdom. This phase 3 trial (ISRCTN98387754) randomizing patients undergoing RNU for UTUC to either adjuvant platinumbased chemotherapy or surveillance, with an estimated enrollment of 345 patients, will guide whether $\mathrm{AC}$ is actually required, but this trial may be limited by inadequate stratification of local or locoregional disease (estimated completion March 2017). In a sys- tematic review and meta-analysis of adjuvant and neoadjuvant chemotherapy for upper tract urothelial carcinoma published in 2014 [29], the authors conclude that there might be a role for cisplatin-based $\mathrm{AC}$ in UTUC patients who have adequate postoperative renal function and high-risk disease (pT3,pN+, positive surgical margins), but insufficient evidence currently exists to recommend routine use of $\mathrm{AC}$ for all UTUC patients. Recently, Kobayashi et al. [30] determined the effect of preoperative chemotherapy (PC) on survival in 55 patients with UTUC clinically involving regional lymph nodes at a single institution between 1991-2013. PC consisted of two-four cycles of cisplatin-containing regimens. The 5-year overall survival rate was significantly higher in the study group than in control group (44\% vs. $12.9 \%$; $\mathrm{p}=0.003$ ). Neoadjuvant chemotherapy appears to be promising, but more trials are needed to confirm its utility.

\section{Open versus laparoscopic/robotic nephroureterectomy}

Radical nephroureterectomy (RNU) with excision of the bladder cuff is the gold standard treatment for UTUC, regardless of the location of the tumor in the upper urinary tract. The RNU procedure must comply with oncological principles, which consist of preventing tumor seeding by avoiding entry into the urinary tract during tumor resection. The laparoscopic RNU [31] has not yet achieved final proof of its safety. There are early reports of retroperitoneal metastatic dissemination and dissemination along the trocar pathway when large tumors were manipulated in a pneumoperitoneal environment [32]. Several precautions [31] must be taken when operating with a pneumoperitoneum because it may increase tumor spillage:

1. Entering the urinary tract should be avoided.

2. Direct contact of the instruments with the tumor should be avoided (we recommend to clip the ureter as soon as posible).

3. Laparoscopic RNU must take place in a closed system. Morcellation of the tumor should be avoided and an endobag is necessary to extract the tumor.

4. The kidney and ureter must be removed en bloc with the bladder cuff.

5. Invasive or large (T3/T4 and/or $\mathrm{N}+/ \mathrm{M}+)$ tumors are contraindications for laparoscopic RNU, until proven otherwise (authors think it depends on surgeon's experience).

Laparoscopic RNU, however, is becoming an established alternative to open RNU at centers with adequate laparoscopic expertise . Recent data showed 
the evidence for equivalent disease free survival (DFS) and CSS between open and laparoscopic RNU, especially in patients with predominantly favorable clinical and pathologic features [33]. The ability to perform LND during laparoscopy is still a major concern, potentially affecting patient staging and possibly survival; however, in a recent study [34], the median node counts between the 2 methods were identical $(n=8)$, and there was no difference in CSS. In addition, the laparoscopic approach appears to be superior to open surgery only with regard to functional outcomes (less lost blood and a shorter hospital stay). Futhermore, in a randomized singlesurgeon trial of 80 patients [35], there was no diference in CSS or metastasis-free survival (MFS) with respect to open $v s$. laparoscopic RNU. When matched for pT3 and high-grade tumors, CSS and MFS were significantly different between the two groups in favour of open surgery, but one possible explanation may be they did not perform LND during laparoscopy. Taken as a whole, these data seem to conclude that the surgeon should proceed as his or her comfort level dictates with regards to laparoscopic or open surgery.

Recently, a multi-institutional series [36] of robotassisted nephroureterectomy (RANU) has been published with respect to the technique and perioperative outcomes. Pathology was pTa in nine patients, pT1 in 14 patients, pT2 in three patients, pT3 in 15 patients, and pT4 in two patients. Lymph node dissection was performed in 22 patients $(51 \%)$ with a mean (range) lymph node count of 11 [4-23]. They conclude that RANU is a feasible alternative to laparoscopic and open techniques. Particular steps of the operation including sutured closure of the cystotomy and regional lymphadenectomy are facilitated with the use of robot-assisted surgery.

\section{Therapeutic benefits of lymphadenectomy in upper tract urothelial carcinoma}

Several papers have investigated the role of LND and nodal status on DFS and CSS (Table 2). Roscigno et al. [18, 19] observed 132 consecutive patients with muscle-invasive UTUC who underwent radical surgery at a single institution. A statistically significant difference in terms of actuarial 5-yr CSS emerged between $\mathrm{pN} 0$ and $\mathrm{pN}+$ and between $\mathrm{pN} 0$ and $\mathrm{pNx}$ patients, but not between $\mathrm{pNx}$ and $\mathrm{pN}+$ patients. This was confirmed at multivariable Cox regression analyses, where nodal status emerged as an independent predictor of both DFS and CSS. In this population, a higher percentage of $\mathrm{pNx}$ patients probably would have had positive nodes if LND had been performed. However, it is likely
Table 1. Primary site of nodal metastases of UTUC

\begin{tabular}{ccc}
\hline Right renal pelvis & $\begin{array}{c}\text { Retrocaval nodes, } \\
\text { hilar and paracaval sites } \\
\text { Upper two thirds of the right ureter } \\
\text { Retrocaval nodes, } \\
\text { hilar and paracaval sites } \\
\text { Interaortocaval sites }\end{array}$ \\
Left renal pelvis & Renal hilum and para-aortic nodes \\
Upper two thirds of the left ureter & Para-aortic nodes \\
\hline $\begin{array}{c}\text { Lower thirds of the ureter } \\
\text { on each side }\end{array}$ & $\begin{array}{c}\text { Pelvic lymph nodes below the aortic } \\
\text { bifurcation (common iliac, obturator } \\
\text { and internal iliac nodes) }\end{array}$ \\
\hline
\end{tabular}

UTUC - Upper Tract Urothelial Carcinoma

that the true rate of node-positive disease has been under-reported because these data are retrospective. The LDN template is likely to have a greater impact on patient survival than the number of lymph nodes removed [37]. Lymph node dissection can be achieved following lymphatic drainage described previously. In a prospective study, Kondo et al. [37] reported patients with pT2 or higher UTUC who underwent template based LND with RNU had higher CSS than a control group (89.8\% vs. $51.7 \%)$. Matin el al. [38] performed a retrospective multi-institutional study in a larger cohort undergoing template disecction, showing right to left as well as cranial migration, and suggesting templates. For example, on the right side, resection of the hilar, paracaval and retrocaval LNs would identify $82.9 \%$ of LN metastases, while adding Interaortocaval LNs would increase that to $95.8 \%$. LND appears to confer a survival benefit in those with high-risk disease. More recent data [38] highlights the reduction in locoregional recurrence when an adequate LND is performed. In the only meta-analysis published in the literature [39] about the effect of lymph node dissection on the outcomes of UTUC, there was a better CSS in patients with muscle invasive UTUC receiving LDN vs. non-LDN (HR: 2.19) and better CSS in pN0 vs. pNx. Of course, we should not forget the WillRogers phenomenon could affect the survival benefit of LND. In oncology, new imaging tools allowed detection of cancer metastases before becoming evident clinically. In consequence, more patients are classified into the more severe metastatic disease stage from the less severe single tumor stage. Such a 'stage migration' resulted in an improved survival of patients in the less and more severe disease stages.

\section{CONCLUSIONS}

Lymphadenectomy has recently attracted considerable interest from urological surgeons and might 
CEnTRAL EURopean JoURnAl of UROLOGY

Table 2. Reports on staging and therapeutic benefit of lymphadenectomy in upper tract urothelial carcinoma

\begin{tabular}{|c|c|c|c|c|c|c|c|}
\hline Authors & Year & № patients & Template of LND & Staging benefits & Therapeutic benefits & $\begin{array}{c}\text { Subject } \\
\text { benefiting } \\
\text { form LND }\end{array}$ & $\begin{array}{l}\text { Minimum no LN } \\
\text { required }\end{array}$ \\
\hline Komatsu & 1997 & 36 & & $\begin{array}{l}\mathrm{pNO}(25) \\
\mathrm{pN}+(11)\end{array}$ & 5 year CSS $100 \%(\mathrm{NO}), 21 \%(\mathrm{~N}+)$ & & Not determined \\
\hline Miyake & 1998 & 72 & & $\begin{array}{l}\mathrm{pNO}(22) \\
\mathrm{pNx}(37) \\
\mathrm{pN}+(13)\end{array}$ & $\begin{array}{c}5 \text { year CSS } 64 \%(\mathrm{NO}), 50(\mathrm{Nx}) \\
0(\mathrm{~N}+)\end{array}$ & & Not determined \\
\hline Novara & 2007 & 269 & & $\begin{array}{l}\mathrm{pNO}(242) \\
\mathrm{pN}+(27)\end{array}$ & 5 year CSS $82 \%(\mathrm{NO}), 12 \%(\mathrm{~N}+)$ & & Not determined \\
\hline Secin & 2007 & 255 & & $\begin{array}{l}\mathrm{pNO}(89) \\
\mathrm{pN} \times(71) \\
\mathrm{pN}+(24)\end{array}$ & $\begin{array}{c}5 \text { year CSS } 80 \%(N 0), 77 \%(N x), \backslash \\
\text { zs } 35 \%(N+)\end{array}$ & & Not determined \\
\hline Kondo & 2007 & 169 & Clearly described & Not determined & $\begin{array}{c}\text { Yes: complete LND } \\
\text { as an independent for CSS }\end{array}$ & $\geq \mathrm{pT3}$ & Not determined \\
\hline Brausi & 2007 & 83 & Described & Not determined & $\begin{array}{c}\text { Yes: LND } \\
\text { as an Independent for OS }\end{array}$ & $\geq \mathrm{pT} 2$ & Not determined \\
\hline Roscigno & 2008 & 132 & Described & $\begin{array}{l}\text { Yes: CSS } \\
\text { is } p N O>p N x\end{array}$ & $\begin{array}{c}\text { Yes; } \mathrm{LN} \geq 6 \\
\text { as an Independent for CSS }\end{array}$ & $\geq \mathrm{pT} 2$ & 6 \\
\hline Roscigno & 2009 & 1130 & Not well described & $\begin{array}{l}\text { Yes: CSS } \\
\text { is } p N O>p N x\end{array}$ & Not determined & $\geq \mathrm{pT} 2$ & Not determined \\
\hline Roscigno & 2009 & 552 & Not well described & Not determined & $\begin{array}{c}\text { Yes: } \mathrm{LN} \geq 8 \\
\text { as an Independent for CSS }\end{array}$ & pNO & 8 \\
\hline Kondo & 2010 & 209 & Clearly described & Not determined & $\begin{array}{l}\text { Yes: Complete LND } \\
\text { as Independent for recurrence }\end{array}$ & $\geq \mathrm{pT} 2$ & $\begin{array}{l}\text { Not a significant } \\
\text { factor }\end{array}$ \\
\hline Abe & 2010 & 293 & Not well described & $\begin{array}{l}\text { Yes: DFS } \\
\text { is } p N O>p N x\end{array}$ & $\begin{array}{l}\text { Yes: LDN } \\
\text { as Independent for recurrence }\end{array}$ & $\geq \mathrm{pT} 2$ & Not determined \\
\hline Lughezzani & 2010 & 2824 & Not described & $\begin{array}{l}\text { Yes: CSS } \\
\text { is } p N+<p N x\end{array}$ & $\mathrm{No}$; $\mathrm{CSS}$ is $\mathrm{pNo}=\mathrm{pNx}$ & Any $T$ & Not determined \\
\hline Burger & 2011 & 785 & Not well described & $\begin{array}{l}\text { Yes: CSS } \\
\text { is } p N+<p N x\end{array}$ & No; CSS is $p N o=p N x$ & Any $T$ & Not determined \\
\hline Kondo & 2014 & 77 & Yes clearly described & $\begin{array}{l}\operatorname{CSS}(89.8 \% \text { vs. } \\
51.7 \%, p=0.01)\end{array}$ & $\begin{array}{l}\text { OS ( } 86.1 \% \text { vs. } 48 \%, p=0.01) \\
\text { DFS } 77.8 \% \text { vs. } 50 \%, p=0.06\end{array}$ & $\geq \mathrm{pT} 2$ & $\begin{array}{l}\text { Not a significant } \\
\text { factor }\end{array}$ \\
\hline
\end{tabular}

CSS - cancer specific survival, LN - lymph nodes, LND - lymphadenectomy, DFS - disease free survival, OS - overall survival

have a potential role in improving the oncological outcome in patients with urothelial carcinoma. LND ideally improves disease staging; thereby, we need to find the way to identify the patients who could really benefit from adjuvant systemic theraphy. Template-based LND with RNU for high risk disease is gaining support based on accumulating retrospective data and supports its utility as a potencially therapeutic maneuver [40]. RNU [41] is still the gold standard treatment for UTUC but minimal invasive procedures such as laparoscopic RNU and RANU are becoming more employed in recent years and should be used by expert hands. Therapeutic benefits of LND and nodal status on DFS and CSS remains controversial, although most of the data comes from retrospective studies, we encourage performing well designed, prospective and multicentre studies to clarify this in the coming years.

\section{CONFLICTS OF INTEREST}

The authors declare no conflicts of interest.

\section{References}

1. Capitanio U, Becker F, Blute ML, et al. Lymph node dissection in renal cell carcinoma. Eur Urol. 2011; 60: 1212-1220.
2. Miki J, Egawa S. The role of lymph node dissection in the management of prostate cancer. Int J Clin Oncol. 2011; 16: 195-202.
3. Kitamura H, Masumori N, Tsukamoto T. Role of lymph node dissection in management of bladder cancer. Int J Clin Oncol. 2011; 16: 179-185. 
4. Roscigno M, Brausi M, Heidenreich A, et al. Lymphadenectomy at the time of nephroureterectomy for upper tract urothelial cancer. Eur Urol. 2011; 60: 776-783.

5. Crispen PL, Breau RH, Allmer C, et al. Lymph node dissection at the time of radical nephrectomy for high-risk clear cell renal cell carcinoma: indications and recommendations for surgical templates. Eur Urol. 2011; 59: 18-23.

6. DiMarco DS, Zincke H, Sebo TJ, Slezak J, Bergstralh EJ, Blute ML. The extent of lymphadenectomy for PTXNO prostate cancer does not affect prostate cancer outcome in the prostate specific antigen era. J Urol. 2005; 173: 1121-1125.

7. Briganti A, Blute ML, Eastham JH, et al. Pelvic lymph node dissection in prostate cancer. Eur Urol. 2009; 55: 1251-1265.

8. Capitanio U, Jeldres C, Patard JJ, et al. Stage-specific effect of nodal metastases on survival in patients with nonmetastatic renal cell carcinoma. BJU Int. 2009; 103: 33-37.

9. Stein JP, Lieskovsky G, Cote R, et al. Radical cystectomy in the treatment of invasive bladder cancer: long-term results in 1,054 patients. J Clin Oncol. 2001; 19: 666-675.

10. Zigeuner R, Pummer K. Urothelial carcinoma of the upper urinary tract: surgical approach and prognostic factors. Eur Urol. 2008; 53: 720-731.

11. Ozsahin M, Zouhair A, Villa S, et al. Prognostic factors in urothelial renal pelvis and ureter tumors: a multicentre Rare Cancer Network study. Eur J Cancer. 1999; 35: 738-743.

12. Russo P. Editorial comment on 'Assessment of the mínimum number of lymph nodes needed to detect lymph node invasion at radical nephroureterectomy in patients with upper tract urothelial cancer'. Urology. 2009; 74: 1075-1076.

13. Batata MA, Whitmore WF, Hilaris BS, Tokita N, Grabstald H. Primary carcinoma of the ureter: a prognostic study. Cancer. 1975; 35: 1626-1632.

14. McCarron JP Jr, Chasko SB, Gray GF Jr. Systematic mapping of nephroureterectomy specimens removed for urothelial cancer: pathological findings and clinical correlations. J Urol. 1982; 128: 243-246.

15. Akaza H, Koiso K, Niijima T. Clinical evaluation of urothelial tumors of the renal pelvis and ureter based on a new classification system. Cancer. 1987; 59: 1369-1375.

16. Komatsu H, Tanabe N, Kubodera S, Maezawa $\mathrm{H}$, Ueno $\mathrm{A}$. The role of lymphadenectomy in the treatment of transitional cell carcinoma of the upper urinary tract. J Urol. 1997; 157: 1622-1624.

17. Miyake H, Hara I, Gohji K, Arakawa S, Kamidono S. The significance of lymphadenectomy in transitional cell carcinoma of the upper urinary tract. Br J Urol. 1998; 82: 494-498.

18. Roscigno M, Shariat SF, Margulis V, et al. Impact of lymph node dissection on cancer specific survival in patients with upper tract urothelial carcinoma treated with radical nephroureterectomy. J Urol. 2009; 181: 2482-2489.

19. Roscigno M, Shariat SF, Margulis V, et al. The extent of lymphadenectomy seems to be associated with better survival in patients with nonmetastatic upper-tract urothelial carcinoma: how many lymph nodes should be removed? Eur Urol. 2009; 56: 512-519.

20. Kondo T, Nakawaza H, Ito F, et al. Primary site and incidence of lymph node metastases in urothelial carcinoma of upper urinary tract. Urology. 2007; 69: 265-269.

21. Abe T, Shinohara N, Harabayashi T, et al. The role of lymph node disecction in the treatment of upper urinary tract cancer: a multi-institutional study. BJU Int. 2008; 102: 576-580.

22. Song YS, Cho JS, Cho KS, et al. Efficacy of adjuvant gemcitabine cisplatin chemotherapy: a comparative study between locally advanced transitional cell carcinoma of the bladder and upper urinary tract. Urol Int. 2010; 85: 47-51.

23. Hellenthal NJ, Shariat SF, Margulis V, et al. Adjuvant chemotherapy for high risk upper tract urothelial carcinoma: results from the upper tract urothelial carcinoma collaboration. J Urol. 2009; 182: 900-906.

24. O'Donnell PH, Stadler WM. The role of chemotherapy in upper tract urothelial carcinoma. Adv Urol. 2009: 419028.

25. Kim TS, Oh JH, Rhew HY. The efficacy of adjuvant chemotherapy for locally advanced upper tract urothelial cell carcinoma. J Cancer. 2013; 4: 686-690.
26. Youssef RF, Shariat SF, Lotan Y, et al. Upper urinary tract urothelial carcinoma with loco-regional nodal metastases: insights from the upper tract urothelial carcinoma collaboration. BJU Int. 2011; 108: 1286-1291.

27. Matin SF, Margulis V, Kamat A, et al. Incidence of downstaging and complete remission after neoadjuvant chemotherapy for high-risk upper tract transitional cell carcinoma. Cancer. 2010; 116: 3127-3134.

28. Birtle AJ, Lewis R, Johnson M, et al. Time to define an international standard of postoperative care for resected upper urinary tract transitional cell carcinoma (TCC)- opening of the perioperative chemotherapy versus surveillance in upper tract urothelial cancer (POUT) Trial. BJU Int. 2012; 110: 919-921.

29. Leow JJ, Martin-Doyle W, Fay AP, et al. A Systematic Review and Meta-analysis of Adyuvant and Neoadyuvant Chemotherapy for Upper Tract Urothelial Carcinoma. Eur Urol. 2014; 66: 529-541.

30. Kobayashi K, Saito T, Kitamura Y, et al. Effect of preoperative chemotherapy on survival of patients with upper urinary tract urothelial carcinoma clinically involving regional lymph nodes. Int J Urol. 2016; 23: 153-158.

31. Rouprêt $M$, Babjuk $M$, Compérat $E$, et al. European Association of Urology Guidelines on Upper Urinary Tract Urothelial Cell Carcinoma: 2015 Update. Eur Urol 2015; 68: 868-879.

32. Rouprêt M, Smyth G, Irani J, et al. Oncological risk of laparoscopic surgery in urothelial carcinomas. World J Urol. 2009; 27: 81-88.

33. Capitanio U, Shariat SF, Isbarn H, et al. Comparison of oncologic outcomes for open and laparoscopic nephroureterectomy: a multiinstitutional analysis of 1249 cases. Eur Urol. 2009; 56: 1-9.

34. Favaretto RL, Shariat SF, Chade DC, et al. Comparison between laparoscopic and open radical nephroureterectomy in a contemporary group of patients: are recurrence and disease-specific survival associated with surgical technique? Eur Urol. 2010; 58: 645-651.

35. Simone G, Papalia R, Guaglianone S, et al. Laparoscopic versus open nephroureterectomy: perioperative and oncologic outcomes from a randomised prospective study. Eur Urol. 2009; 56: 520-526. 
36. Pugh J, Parekattil S, Willis D, Stifelman M, Hemal A, Su LM. Perioperative outcomes of robot-assisted nephroureterectomy for upper urinary tract urothelial carcinoma: a multi-institutional series. BJU Int. 2013; 112: 295-300.

37. Kondo $\mathrm{T}$, Hashimoto $\mathrm{Y}$, Kobayashi $\mathrm{H}$, et al. Template-based lymphadenectomy in urothelial carcinoma of the upper urinary tract: impact on patient survival. Int J Urol. 2010; 17: 848-854.
38. Matin SF, Sfakianos JP, Espiritu PN, et al. Patterns of lymphatic metastases in upper tract urothelial carcinoma and proposed disecction templates. J Urol. 2015; 194: 1567-1574.

39. Yang $D$, Chen $Q$, Song $X$, et al. Effect of lymph node dissection on the outcomes of upper tract urothelial carcinomas: a metaanalysis. Expert Rev Anticancer Ther. 2014; 14: 667-675.
40. Rao S. Mandalapu, Surena F. Martin. Contemporary Evaluation and Management of Upper Tract Urothelial Cancer. Urology. 2016; 94: 17-24.

41. Seisen T, Granger B, Colin P, et al. A Systematic Review and Meta-analysis of Clinicopathologic Factors Linked to Intravesical Recurrence After Radical Nephroureterectomy to Treat Upper Tract Urothelial Carcinoma. Eur Urol. 2015; 67: 1122-1133. 